\title{
Urgences
}

\section{Les carnets de voyage du peintre}

\section{Bruno Santerre}

Numéro 24, juillet 1989

Le manuscrit sous l'angle

URI : https://id.erudit.org/iderudit/025537ar

DOI : https://doi.org/10.7202/025537ar

Aller au sommaire du numéro

Éditeur(s)

Urgences

ISSN

0226-9554 (imprimé)

1927-3924 (numérique)

Découvrir la revue

Citer ce document

Santerre, B. (1989). Les carnets de voyage du peintre. Urgences, (24), 103-113. https://doi.org/10.7202/025537ar d'utilisation que vous pouvez consulter en ligne.

https://apropos.erudit.org/fr/usagers/politique-dutilisation/ 


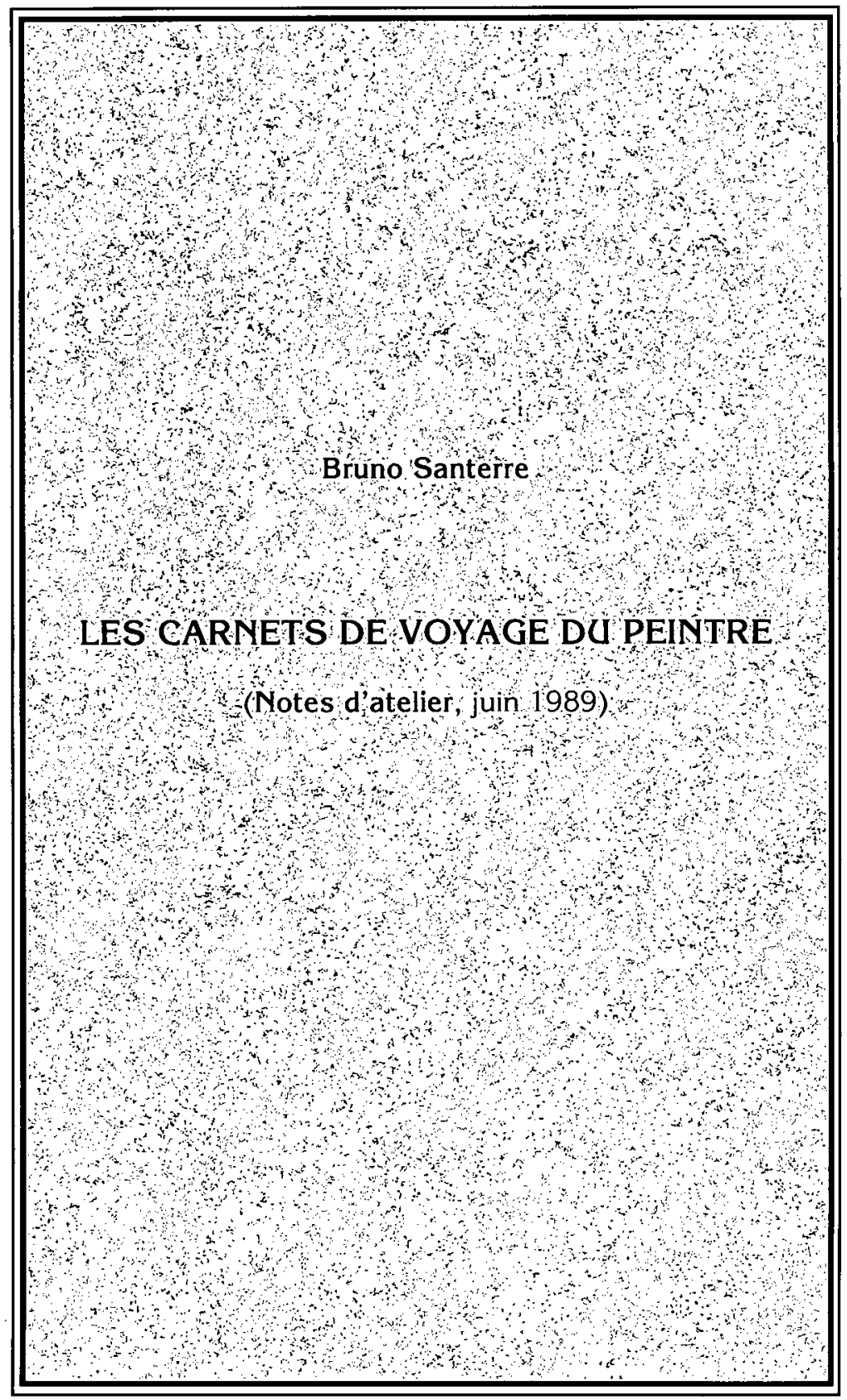


Dans ce double mouvement, de se perdre et de se retrouver, la photographie ressemble étrangement au voyage où il s'agit souvent de se perdre un peu de vue, de se dissoudre, de s'éparpiller tout en se concentrant, d'une certaine façon, dans le fait même de voir: "En voyage, écrit Francis Ponge, il y a voir, en voyage voir est venu et il s'en est fallu de peu, sans doute, que voyager fut dit de l'action même de voir."

\section{Alain Bergala, Les absences du photographe}

A l'occasion d'une escale le peintre vide ses poches, étale leur contenu sur la table, prend un cahier et y colle des fragments d'images et de textes qu'il a amassés au gré de ses errances et de ses rencontres. Il y collectionne ces objets, ces quelques traces, témoins de lieux et de réflexions qui furent déterminants au cours de son itinéraire.

Après avoir répertorié ces quelques objets, il constate jusqu'à quel point il peut retrouver en chacun d'eux des évocations de toutes sortes, de multiples échos provenant de lieux en apparence séparés mais s'échangeant néanmoins un flot continu d'informations...

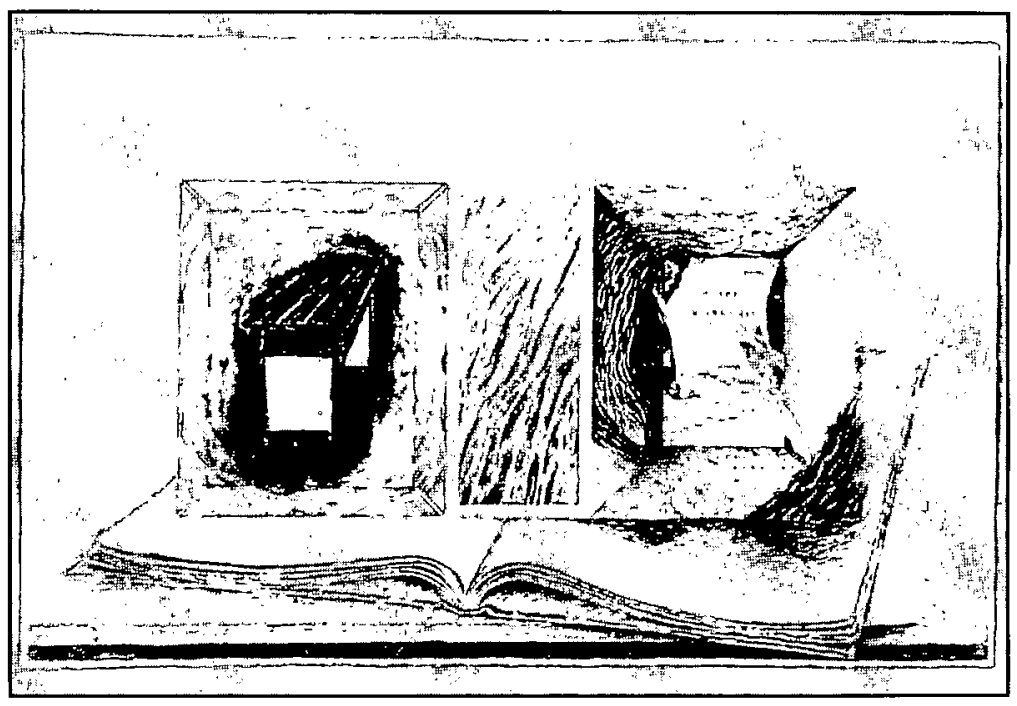

Les carnets de voyage, no. 2 , 1989, techniques mixtes, $66 \times 102 \mathrm{~cm}$. 


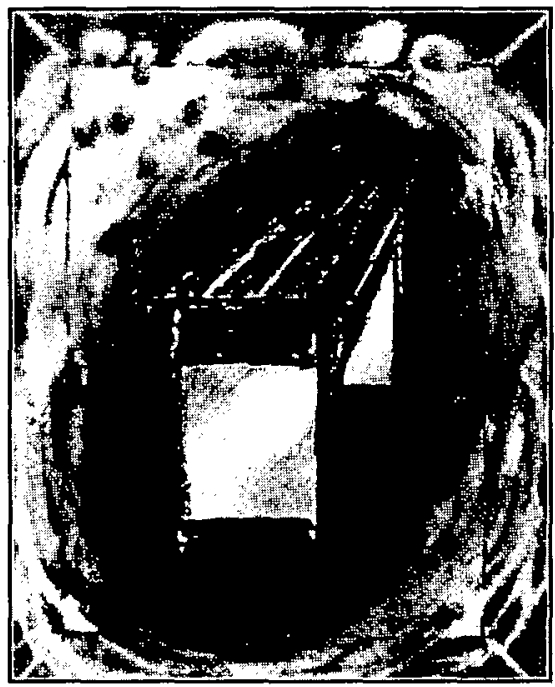

1. Un dessin de table, extrait d'un tableau

La table...

comme lieu de la communication; comme lieu de la rencontre et de l'échange entre le réel et le représenté.

Un objet possédant des qualités formelles d'ouverture et de stabilité. Un objet qui permet de nombreuses permutations dans le plan du tableau, par rotation, rabattement ou translation.

Un objet qu'on peut "sans subterfuges, adapter à la surface du tableau" ${ }^{2}$ et qui peut, par divers procédés picturaux et métaphoriques, aisément se confondre avec le tableau.

Un objet construit par stratification de matières, dissimulant de nombreux secrets à l'intérieur de lui-même.

Une surface plane, lieu de la transformation des matières et de la fabrication des fictions.

Le tableau...

comme lieu où se joue la représentation; comme lieu qui se joue de la représentation.

Un objet qui, tout en demeurant continuellement insaisissable, s'amuse à se confondre avec la surface du mur.

Une construction à mi-chemin entre l'objet et l'image, se situant à la fois dans un espace très rapproché de nous et, à d'autres moments, dans un espace illusoire nous semblant très éloigné, hors de notre portée.

Une surface où les objets se rencontrent, se reflètent l'un dans l'autre et cherchent à se laver mutuellement de leurs ombres.

Un lieu où toute transformation ou distorsion du réel est possible. 


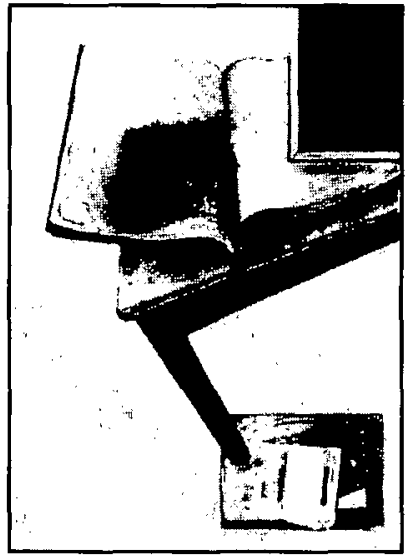

Il n'y a pas de différence entre ce dont un livre parle et la manière dont il est fait. [...] En tant qu'agencement, il est seulement lui-même en connexion avec d'autres agencements, par rapport à d'autres corps sans organes. On ne demandera jamais ce que veut dire un livre, signifié ou signifiant, on ne cherchera rien à comprendre dans un livre, on se demandera avec quoi il fonctionne, en connexion de quoi il fait ou non passer des intensités, dans quelles multiplicités il introduit et métamorphose la sienne, avec quels corps sans organes il fait lui-même converger le sien.

Gilles Deleuze et Félix Guattari, Mille plateaux ${ }^{3}$

\section{Un livre (ou bien ne s'agirait-il pas du dessin d'un livre?)}

\section{Le livre...}

comme lieu de l'inscription du langage et de l'accumulation des connaissances.

Lieu de la découverte et du doute, du récit et de l'invention.

Un objet ouvert, à la fois opaque et transparent, qui nous laisse voir son contenu, mais qui ne nous révèle qu'une partie de ses secrets.

Un objet construit par la stratification, possédant une masse, une densité créée par la superposition d'une grande quantité de feuilles de papier fragiles et translucides qui prennent une opacité par l'addition, par la reliure.

Un objet à la fois vulnérable et durable.

Un objet composé d'une double surface (elle-même composée d'une multitude de surfaces...), de deux plans distincts se superposant en pivotant et s'articulant autour d'un axe de rotation. Sur ces deux plans qui se font face: des miroirs se réfléchissant l'un dans l'autre et s'échangeant des informations.

Par ses opérations fréquentes de rabattement de plans, d'ouverture et de fermeture, de révélation des secrets ou d'occultation de ceux-ci, ma peinture se propose comme livre, le tableau et la table émergeant de ce livre. 


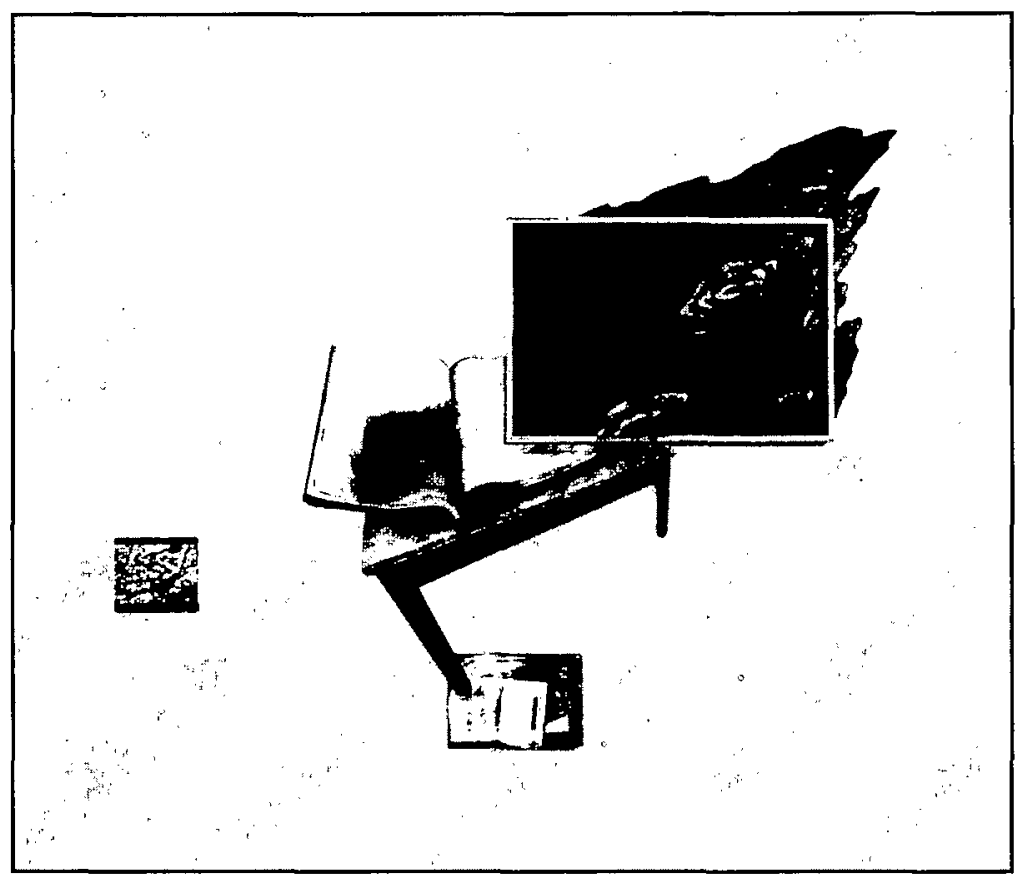

Le spéculaire et l'insondable, 1989 , techniques mixtes, $130 \mathrm{~cm}$. $140 \mathrm{~cm}$. 


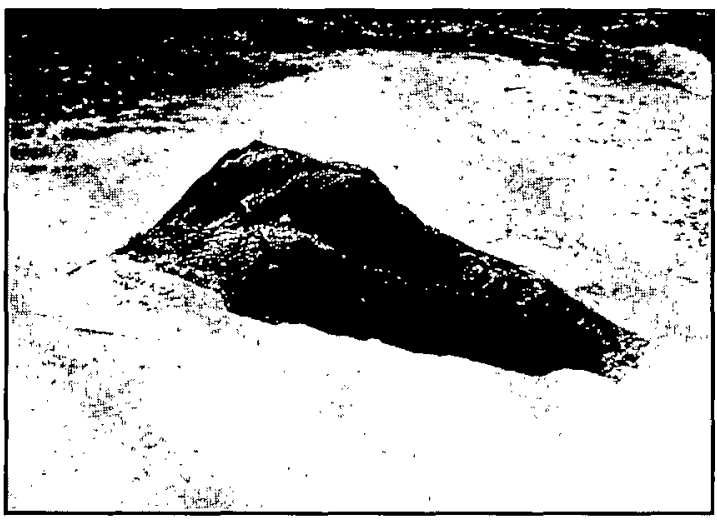

3. Un éclat de rocher, tiré des eaux tumultueuses d'une rivière (ou estce la cime d'une montagne émergeant d'un nuage?)

La pierre ...

Un objet composé de strates tout comme le sont le livre, la table et le tableau.

Ces stratifications recèlent sans doute de multiples secrets: cette pierre ayant la capacité de fixer dans sa matière - comme le fait la photographie - des empreintes de particules du réel, les sauvant ainsi d'une inévitable disparition.

Un objet créé par densification de la matière: des îlots se formant par sédimentation à partir d'éléments en suspension dans l'eau. Cette pierre prend forme - comme dans le cas de la peinture - à partir d'une concentration d'éléments de toutes origines qui résistent momentanément à la dissolution et à l'érosion du temps.

Un objet qui, par son apparente solidité et son immobilité, concentre le temps et l'espace en un point à partir duquel la mobilité et la fugacité des autres objets (devenus signes dans la peinture) nous semblent plus évidentes.

Ce rocher (cette montagne?), en constant échange de qualités de matière avec l'eau (le nuage?), vient nous rappeler que, dans la pensée chinoise:

"L'idée de transformation est fondée sur la conviction que, malgré l'apparente opposition entre les deux entités, celles-ci ont une relation de devenir réciproque. Chacune est, en effet, perçue comme un état sans cesse attiré par l'autre qui lui est complémentaire. ${ }^{4}$

La peinture paysagiste se nommant en Chine "peinture de Montagne et d'Eaun et se proposant comme un miroir de la Nature et de l'Homme inscrit dans celle-ci et comme un lieu où l'on peut fixer momentanément les états passagers de la matière, il devient intéressant alors de tenter un rapprochement entre la peinture utilisant ces signes et le domaine du photographique. 


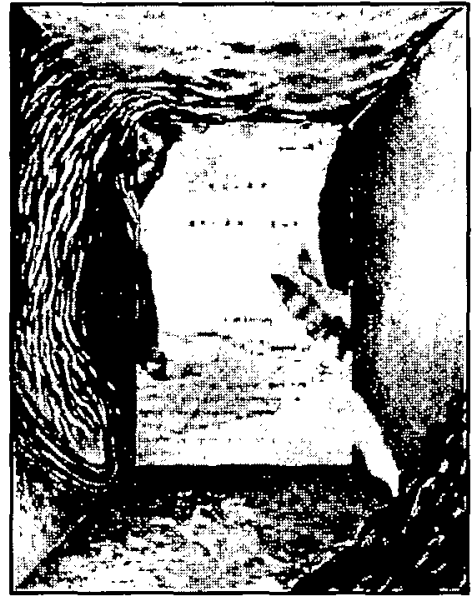

Si la carte s'oppose au calque [associé à la photo par les auteurs] c'est qu'elle est toute entière tournée vers une expérimentation en prise sur le réel. [...] Elle concourt à la connexion des champs, $[\ldots]$ à leur ouverture maximum sur un plan de consistance. Elle fait elle-même partie du rhizome. La carte est ouverte, elle est connectable dans toutes ses dimensions, démontable, renversable, susceptible de recevoir constamment des modifications. Elle peut être déchirée, renversée, s'adapter à des montages de toute nature, être mise en chantier par un individu, un groupe, une formation sociale. On peut la dessiner sur un mur, la concevoir comme une oeuvre d'art, la construire comme une action politique ou comme une méditation. C'est peut-être un des caractères les plus importants du rhizome, d'être toujours à entrées multiples.

Gilles Deleuze et Félix Guattari, Mille plateaux ${ }^{5}$

\section{Un fragment de carte géographique, déchiré puis recollé}

\section{La peinture comme carte?}

Et si je tentais de trouver le trait d'union entre l'espace tactile et sensible de la topologie et celui, visuel et perspectiviste, de la Renaissance, la cartographie pourrait peut-être servir de lien entre ces deux façons de concevoir la représentation spatiale.

On sait que Ptolémée a fait le rapprochement entre la peinture et la cartographie. Il note que, dans ces deux cas, il s'agit de "construire l'image d'un ensemble donné en respectant les justes proportions de ses éléments" ${ }^{6}$. En ce sens la topographie, la cartographie et la perspective apparaissent comme des branches d'une science générale de la représentation spatiale. Donc, en m'appuyant sur ces données, je pense à:

Produire une oeuvre qui se propose comme un point de vue, un regard sur le monde qui ne soit pas unique et privilégié.

Ramener un tracé de mon itinéraire à travers ce monde des choses et me construire une image - la plus précise possible - de ces espaces traversés tout en acceptant que cette image pourra ne pas être tout à fait fidèle, qu'elle pourra comporter des zones d'ombre. Cette image se trouvera alors chargée d'un certain mystère et sera d'autant plus évocatrice, comme l'étaient, par exemple, les cartes ramenées par les explorateurs des temps anciens.

Inventer une topographie de l'itinéraire de l'artiste voyageant dans le monde des choses en considérant la carte - et la peinture - comme un rhizome en connexion constante avec une constellation de lieux... 


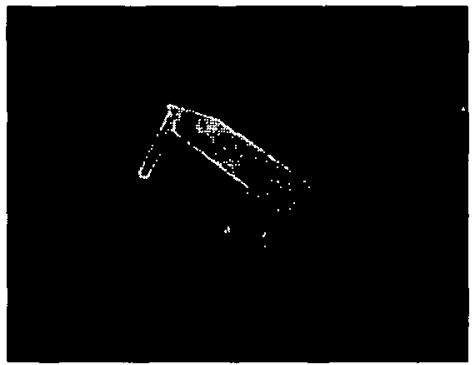

Et si à l'origine de la peinture on retrouvait tout le domaine du photographique ? ${ }^{7}$

La peinture ne pourrait-elle pas alors être considérée comme une surface sensible sur laquelle l'artiste tente de fixer les états de la matière et du réel en perpétuelle transformation? ...comme une surface sur laquelle il s'évertue, pas toujours avec une parfaite exactitude (fort heureusement d'ailleurs), à retenir des ombres fugitives et ades petits fragments du monde qui, la plupart du temps, se perdent et sombrent dans l'impalpablen ${ }^{8}$.

\section{Une photographie, légèrement voilée de suie}

\section{La peinture comme photographie?}

Mon projet à partir de là sera donc de:

Considérer la surface de ma peinture comme reflet ou comme miroir comme daguerréotype? -, c'est-à-dire comme une surface sensible capable de fixer momentanément les états passagers de la matière et les ombres fugitives du réel.

Chercher à établir des relations entre cette surface réfléchissante et les profondeurs insondables des zones ombragées de l'image; entre cette surface miroitante, ayant le pouvoir de renverser le réel, et le sens caché derrière les signes structurant l'image.

Photographier la rivière mais être limité à n'en saisir que la surface réfléchissante et, par là, réaliser l'impossibilité de saisir le réel dans son déroulement ininterrompu.

Répondre à cette déception en édifiant des fictions au moyen desquelles je parviendrai peut-être à éclairer cette vue que j'ai du monde, à rendre ma vision plus nette.

Naviguer à travers les reflets et aborder le sublime, l'indicible, le flou... 


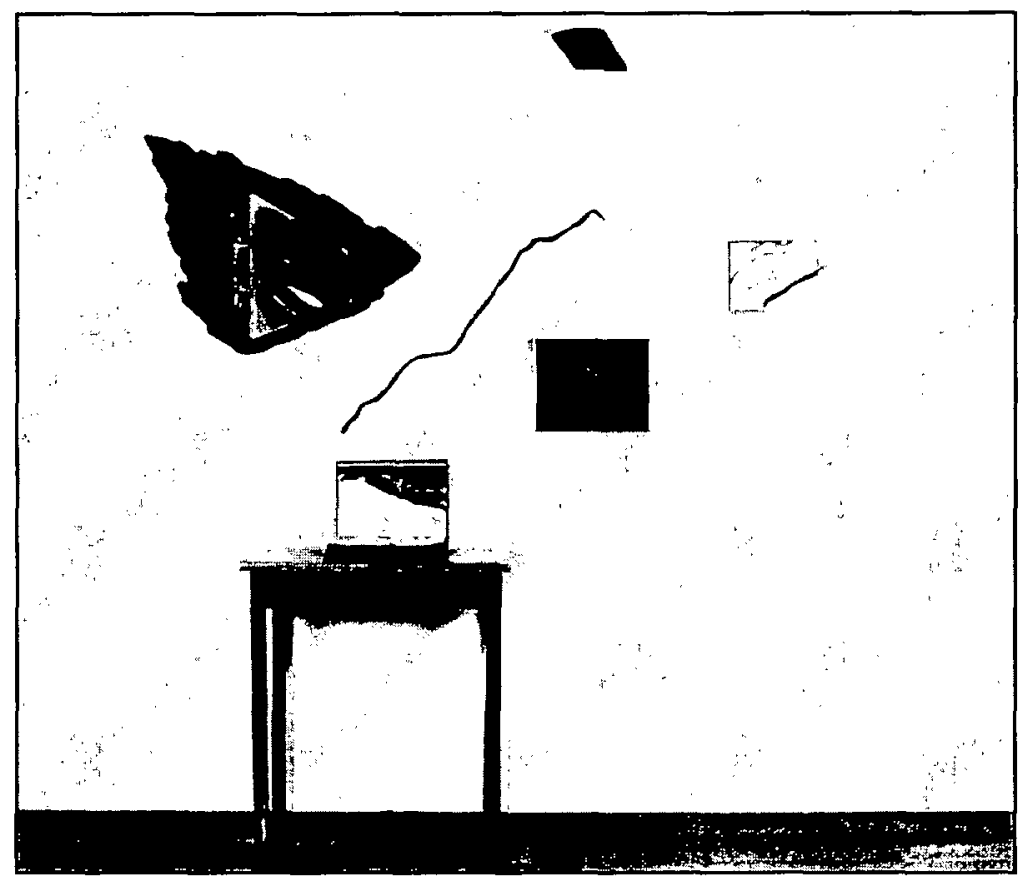

Le proche et le lointain, 1989 , techniques mixtes, $200 \mathrm{~cm} . \times 190 \mathrm{~cm} . \times 40 \mathrm{~cm}$. 
C'est donc de cela qu'il s'agit dans toute photographie: trancher dans le vif pour perpétuerlemort. D'un coup de bistouri, décapiter le temps, prélever l'instant et l'embaumer. Sous (sur) des bandelettes de pellicule transparente, bien à plat et bien en vue, afin de le conserver et le préserver de sa propre perte. Le dérober pour mieux l'enrober, et le donner à voir à jamais. L'arracher à la fuite ininterrompue quil'aurait amené à la dissolution une fois pour toutes dans ses apparences arrêtées. Et ainsi, d'une certaine façon, - voilà le paradoxal enjeu - le sauver de la disparition en le faisant disparaitre.

Philippe Dubois, L'acte photographique ${ }^{9}$

\section{La photographie comme résidu du réel ?}

Penser la peinture comme photographie serait peut-être une façon de dire l'absence et, paradoxalement, de dire la présence de réel dans l'oeuvre d'art - la photographie étant à la fois une pseudo-présence et une marque de l'absence.

Dans le cas du passage de la peinture au photogramme et à la photographie la distance du signe à son objet est réduite au minimum: on passe de l'ordre de la métaphore (esthétique de la mimésis, de l'analogie et de la ressemblance) à celui de la métonymie (une esthétique de la trace, du contact et de la contiguïté référentielle). Mais ce que je retrouve comme trace du réel dans la photographie, ne serait-ce pas qu'une ombre fixée - qu'un bruissement - de cette réalité? Ce serait un peu comme si, lorsque je m'approche trop près de l'objet pour le saisir, je le brûle et que tout ce qui me reste au bout des doigts ne serait qu'une trace de suie ou qu'un peu de cendre, résidus d'un réel évanoui.

Ces résidus de réel constitueront alors l'amorce des procédés fictionnels qui seront mis en oeuvre dans ma peinture avec toute cette relation au flou, à l'indicible et au sublime que cela suppose... 


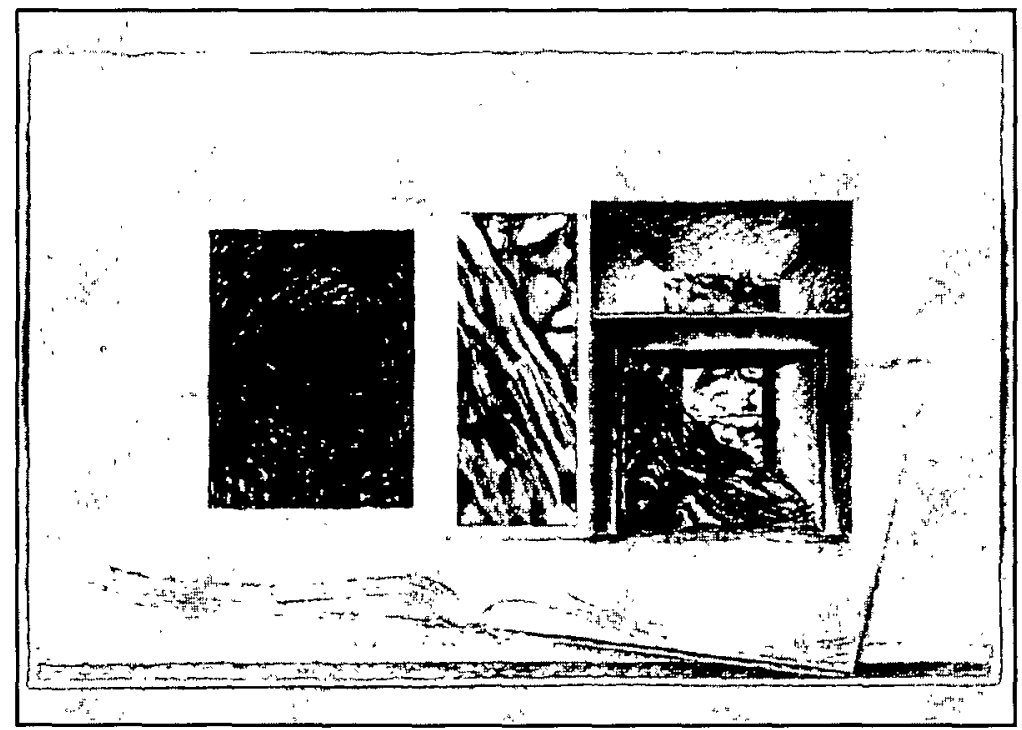

Les carnets de voyage, no.1, 1989, techniques mixtes, $66 \mathrm{~cm}$. $102 \mathrm{~cm}$.

...mais voilà que je me rends compte que ce voyage s'est entièrement déroulé dans mon atelier - ce lieu de connexion des espaces et de la mémoire -, que je suis demeuré immobile tout ce temps et que ce déplacement s'est réaliséà travers les multiples lieux de ma pensée, de la fiction, de l'art et de son histoire.

Je prends alors conscience que j'ai pendant tout ce temps - tel un rhizome sillonnant à travers les sédiments et les strates du sol-parcouru mes notes de lecture et observé mes oeuures récentes, passant d'un plateau à l'autre en cherchant à établir des relations entre ceux-ci.

Je réalise en fin de compte qu'à la suite de ce périple à travers les archipels de ma pensée je pourrai sans doute profiter de cette nouvelle expérience. Elle m'aura amené à formuler de nouvelles questions qui $m$ 'accompagneront dans mes prochaines errances...

1. Alain Bergala, Les absences du photographe, Parıs, Libératıon/Ed. de l'Étoile, 1981, p. 64.

2. Albert Gleızes et Jean Metzinger, Du "cublsme», Paris, Ed. Présence, 1947.

3. Gilles Deleuze et Félix Guattari, Mille plateaux, Paris, Minut, 1980, p. 10.

4. François Cheng, VIde et plein. Le langage pictural chinois, Paris, Seuil, 1979, p.59.

5. Gilles Deleuze et Félix Guattari, Mille plateaux, p.20.

6. Cité par Pierre Thuillier, *Espace et perspective au Quattrocento., La Recherche, Paris, no 160, novembre 1984, p.1395

7. Philippe Dubois, dans L'acte photographique (Paris, Ed. Nathan et Bruxelles, Ed. Labor, 1983), avance cette théorie en farsant référence au mythe fondateur de la peinture tel que relaté par Pline. Celui-ci écrit, dans le livre 35 de son Historia naturalis qui porte sur l'nhistoire. de la penture, que la peinture est littéralement née dans l'ombre. En effet, selon lui, presque tous les commentateurs s'accordent pour dire que la peinture est née de ce qu'son commença par cerner le contour de l'ombre humainex.

8. Lisanne Nadeau, :De quelques territorres en dériven, dans le cahier de présentation de l'exposition Les paysages Indicibles de Bruno Santerre, Musée régional de Rimouski, 1989.

9. Philippe Dubois, L'acte photographique, p.161. 